\title{
A cloud-service Home Health Monitoring System for the Older People Based on WBANs
}

\author{
Lin Wang ${ }^{1, a}$, Jianjun $\mathrm{Yi}^{1, \mathrm{~b}}$, Xiaomin $\mathrm{Zhu}^{1, \mathrm{c}}$ and $\mathrm{Yi} \mathrm{Li}^{1, \mathrm{~d}}$ \\ ${ }^{1}$ Departement of Mechanical Engineering, East China University of Science and Technology, \\ Shanghai 200237, China \\ alinwang4245@163.com, bjyyi@ecust.edu.cn, czxmin4236@163.com, dy_ecust@sina.com
}

\begin{abstract}
Keywords: Home heath monitoring, WBANs, Physiological parameters, Remote cloud server. Abstract. This paper proposes a cloud-service home health monitoring system based on WBANs for tracking the health status of the older people while supporting their mobility. The system consists of a WBANs physiological parameters acquisition system and a remote cloud server. While the former subsystem is responsible for collecting and transmitting the physiological parameters of the older people. And the latter server integrated WBANs with cloud computing provides users with remote services. In this paper, the specific implementation of WBANs physiological parameters acquisition system and the remote cloud server are described. In addition, to facilitate the older people or their families to learn their health status, a simple android app is developed. Finally, the paper takes ECG and body temperature as an example, carry out two functional validation. The result shows a superior performance of the proposed system.
\end{abstract}

\section{Introduction}

One major challenge to successful aging is the capability to preserve health, or from another perspective to avoid disease. Unfortunately, a large number of the older people are living with chronic disease or disabilities. Considered that routine inspections at the clinics or hospitals are usually costly, inconvenient and daily functioning restricted. Therefore, remote health monitoring becomes inevitable, particularly, there is an urgent need for a modern home health monitoring system for the older people, which has the advantages of simple operation, strong mobility, low cost, convenient to use and so forth. Recent advances in wireless networked systems, intelligent low-power sensors and medical sensors, have led to the development and emergence of new embedded networks in the last years known as Wireless Body Area Networks (WBANs) [1,2,3]. WBANs promise to revolutionize health monitoring by offering continuous and ubiquitous ambulatory health monitoring [4]. However, as the increasing demands from customers and patients, the sensing data is required to be timely processed and the feedback from the doctors is also desirable. Since it requires more network resources, i.e., storage, computation and communication power, it is difficult to achieve these goals only relying on the standalone systems WBANs [5]. In order to find a promising solution to effectively deal with aforementioned limitations, a cloud assisted WBANs flourishes and provides more reliable, real-time, and intelligent health-care services for patients and mobile users [6].

This paper aims to propose an effective solution for home health monitoring system for tracking the health status of the older people while overcome the limitations in current health monitoring systems. Integrating WBANs with cloud computing, a cloud-service home health monitoring system is proposed.

\section{Overall Architecture}

Fig. 1 shows the overall architecture of the proposed system. The system mainly consists of a WBANs physiological parameters acquisition system and a remote cloud server. The WBANs physiological parameters acquisition system is composed of several physiological parameters monitoring nodes and a home gateway node, it is mainly responsible for collecting physiological parameters, such as ECG, body temperature, blood pressure, pulse, blood oxygen and so on, then 
transmitting these data to the remote cloud server. The remote cloud server is responsible for mass storage and analysis of the physiological parameters of the cluster users. According to the analysis results, the corresponding health reports are given, the users and their families have the access to view the reports through smart phones or personal computers. In addition, the remote cloud server can provide users with doctor online services, expert services or hospital linkage service as well

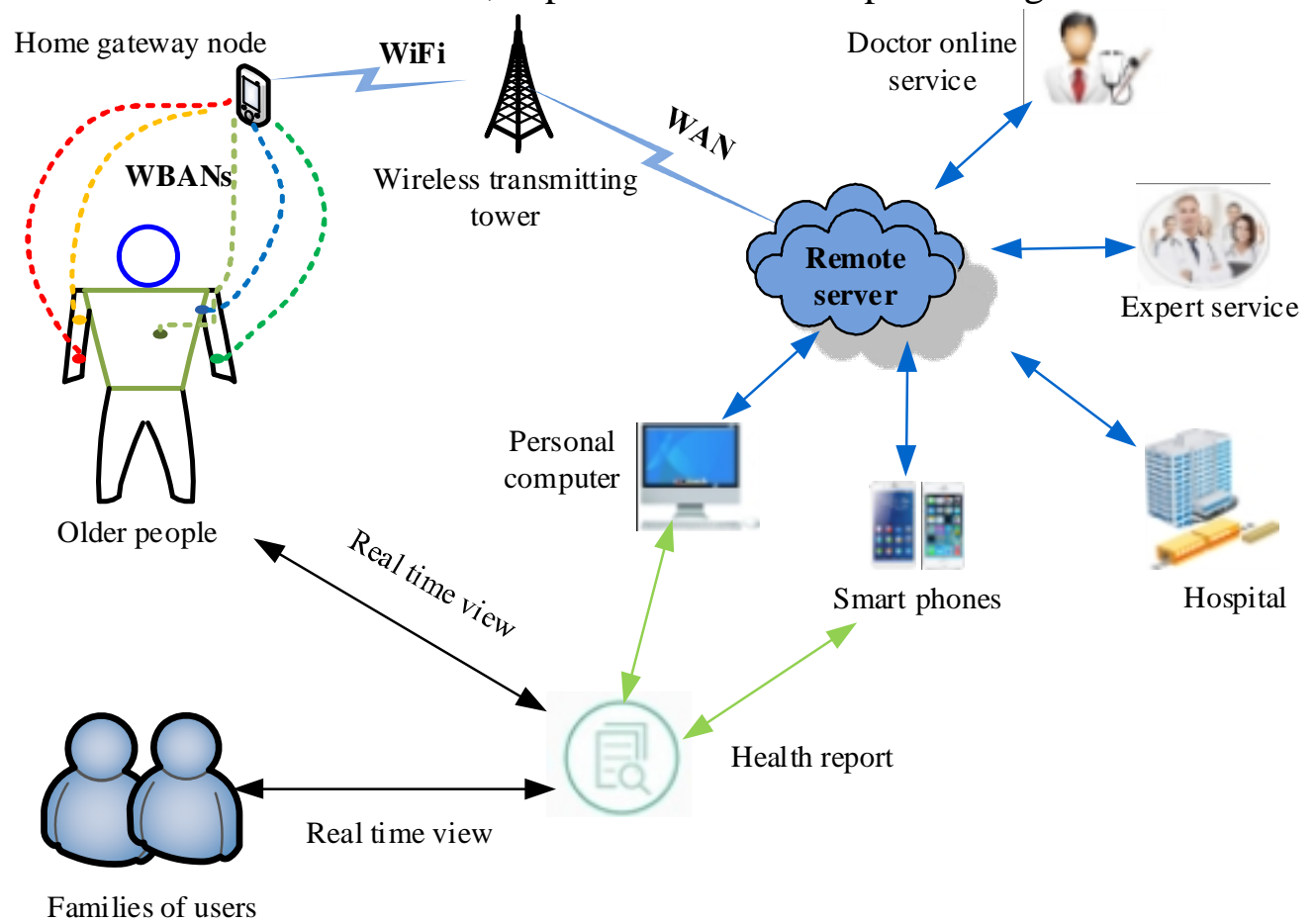

Fig. 1 The overall framework of the proposed system

\section{Implementation of WBANs physiological parameters acquisition system}

\section{WBANs communication mode}

WBANs is a short-range wireless communication networks which established on human body. Currently, there are some common short-range wireless communication network standards including Bluetooth, WiFi, ZigBee and so forth. Table 1 is a comparison of several common wireless communication technologies.

Table 1 The comparison of several common wireless communication technologies

\begin{tabular}{cccc}
\hline Wireless network & Bluetooth & WiFi & ZigBee \\
\hline IEEE standard & IEEE802.15.1 & IEEE802.11 & IEEE802.15.4 \\
Range $(\mathrm{m})$ & $1 \sim 10$ & $10 \sim 100$ & $10 \sim 75$ \\
Network nodes & 7 & 32 & $255 / 65000$ \\
Power & Middle & High & Low \\
consumption & 720 & $11000+$ & $20 \sim 250$ \\
Rate (Kb/s) & Wireless mouse, & Web, images & WSN, monitoring \\
Application & PDA & & Low power, \\
Characteristics & Low cost, convenient & High rates, flexible & Ad-hoc \\
\hline
\end{tabular}

As shown in table 1, Bluetooth is usually applied to wireless mouse, PDA and so forth, but its communication distance is short, generally less than 10 meters, and its protocol is complicated. WiFi is mainly used for web network connection, since it has high data transmission rate, long communication distance, while its power consumption is high and its network architecture is large, so it is not suitable for small low cost network design. While ZigBee has the advantages of close range, low power 
consumption, low cost, ad-hoc network, which is widely used in wireless sensor networks. Therefore, it is easy to find that ZigBee is suitable for deployment in the home use. Accordingly, ZigBee wireless communication mode is adopted in the WBANs. After repeated demonstration, we finally choose CC2530F256 chip as the main control chip of the WBANs nodes in this paper, since this chip is a real SoC (System on Chip) solution used in 2.4GHz IEEE 802.15.4, ZigBee and RF4CE applications. Combined with Z-Stack ${ }^{\mathrm{TM}}$ provided by TI, the WBANs can be established conveniently.

\section{The design of WBANs nodes}

WBANs nodes consist of multiple physiological parameters monitoring nodes and a home gateway node. Fig.2 shows the functional modules of the WBANs nodes and the network diagram. Physiological parameters monitoring nodes contains multiple Zigbee nodes, each of which consists of four parts, they are physiological parameters acquisition sensor, ZigBee module, power amplifier and power module. Among them, the physiological parameter acquisition sensor is responsible for collecting a kind of physiological parameter data, the ZigBee module takes charge of transmitting the physiological parameter data, the power amplifier module undertake to increase the communication distance, and the power module is used to supply power for the above modules. All the Zigbee nodes are independent of each other. This kind of design can not only effectively prevent the collapse of the whole system caused by a sensor, but also greatly reduce the size of the sensor node, which is convenient for the user to wear. The user can freely choose 1 to $n$ nodes, and then collect the 1 to $n$ kinds of physiological parameters.

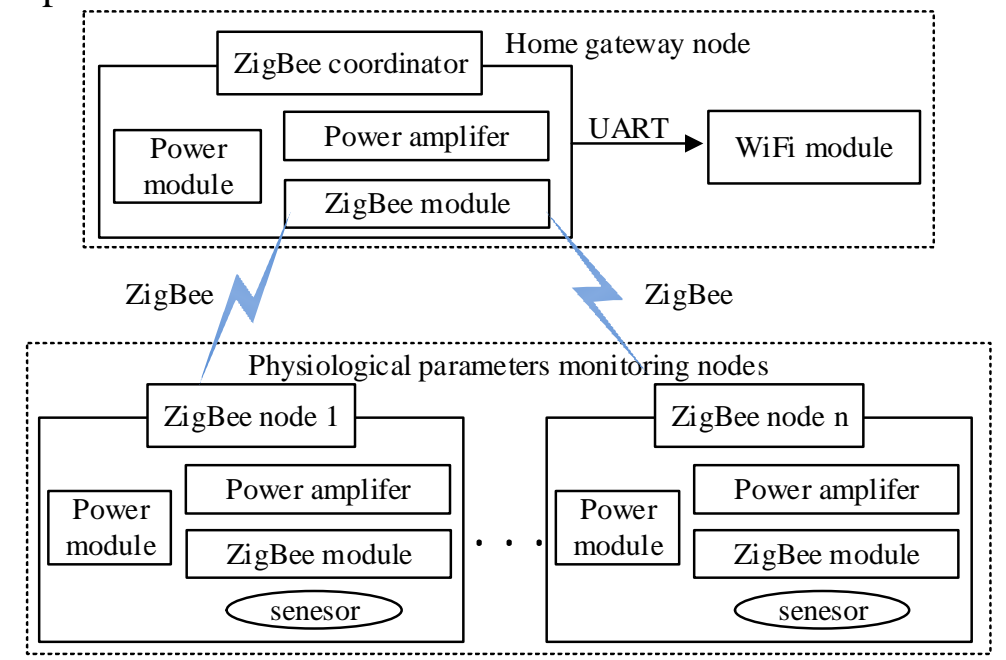

Fig. 2 The function module of the WBANs nodes and the network diagram

The home gateway node is composed of a ZigBee coordinator and a WiFi module. Compared with the ZigBee nodes, the ZigBee coordinator only lacks a physiological parameter acquisition sensor on the composition while other function modules are the same. And it is responsible for the establishment and maintenance of WBANs, and receiving the physiological parameters data from the ZigBee nodes. The WiFi module is responsible for sending the received data to the remote cloud server, so that the following analysis can be processed. The ZigBee coordinator communicates with ZigBee nodes through ZigBee wireless communication protocol, and ZigBee coordinator communicates with the WiFi module through UART (Universal Asynchronous Receiver/Transmitter).

\section{WBANs software design}

The software design flow chart of ZigBee nodes and ZigBee coordinator are shown in Fig.3. In view of ZigBee nodes(Fig.3(a)), when it is started, Zstack will be first initialized, then ZigBee nodes will looking for ZigBee network and join it, if they join the network successfully, they will send the short address to coordinator respectively which is helpful for coordinator to identify and manage them. After that, ZigBee nodes will roll to check whether there is a data request. If there is, the ZigBee nodes will read physiological parameters data from the sensors according to the corresponding request command, and then send these data to ZigBee coordinator node through ZigBee. Similarly, refer to ZigBee coordinator (Fig.3(b)), when it is started, Zstack will be first initialized. Next, the coordinator will build ZigBee network and wait for ZigBee nodes joining it. When ZigBee nodes join the network, 
the coordinator will judge whether it receives the short address of ZigBee nodes, if it received, then it will send data request command to ZigBee nodes automatically, and wait for data return from ZigBee nodes. When coordinator received the return data, it will send the data to WiFi module and finally send the data to remote cloud server.

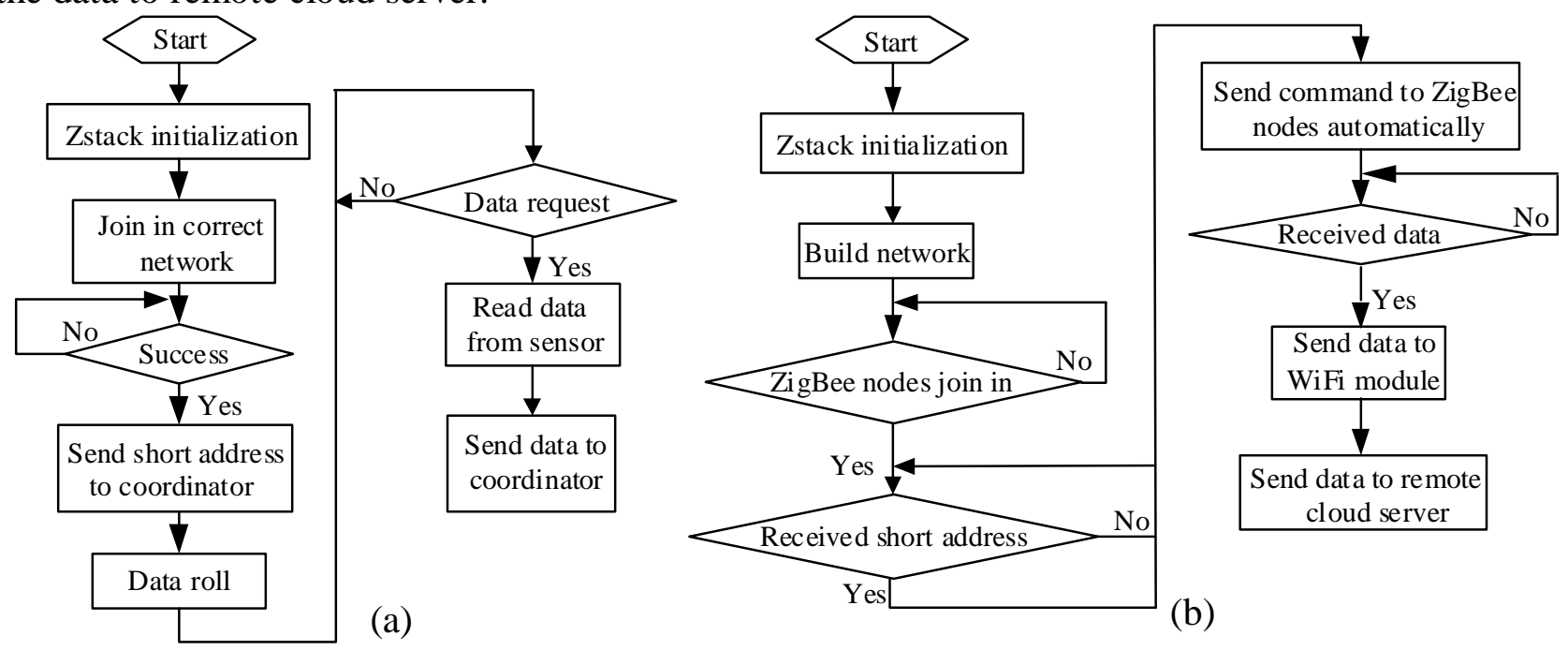

Fig.3 Software design flow chart of WBANs nodes: (a) ZigBee nodes, (b) ZigBee coordinator

\section{The design of remote cloud server and android app}

The most important function of remote cloud server is to complete data storage and analysis. In order to realize the storage of the physiological parameters more conveniently, in this paper we utilize XAMPP to assist the implementation of the remote cloud server. The detailed steps are as follows. Firstly, adopting Apache to implement the construction of the server. Secondly, establishing a TCP link between the WiFi module and the remote cloud server through Java socket. Thirdly, storing the received data into MySQL. Finally, using JDBC (Java Data Base Connectivity) API and PHP background script program to complete the data access. Though above steps, combined with disease diagnostic standards and medical records shared in cloud, basic functions can be achieved.

In order to facilitate the older people and their families to learn their health status anytime and anywhere, a simple android app is developed on mobile phone terminals. The basic development process are as follows: When start the app, users need register and login first, and after that the app will connect to the remote server automatically in the background. If connect successfully, the app will enter the main UI (User Interface). User can select functions, for example physiological parameters data query, health reports and so on, to enter the corresponding UI.

\section{System validation}

Fig.4 shows the prototype of the WBANs physiological parameters acquisition system. In this part, we take ECG and body temperature as an example, carry out two functional validation: mobility test and app test. The mobility mainly affects the association of the ZigBee nodes and the ZigBee coordinator, thus excessive data packets loss may occur as a result of being disassociated due to high mobility. Hence, the mobility test is to examine the data packets loss of the system at different mobile status. And the app test mainly verify the basic functions to facilitate the older people to use this system and learn their health status in real time. 


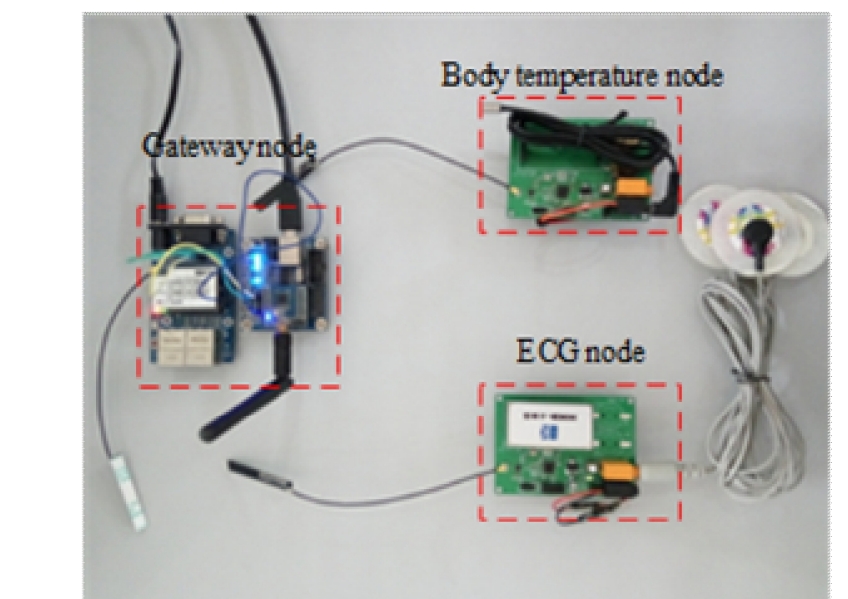

Fig.4 Prototype of the proposed system

In mobility test, as shown in table 1, we assumed three mobility scenarios in which the users moved at different average speed. Simultaneously, in terms of each mobility status we set three kinds of keeping time. In each scenario, we checked the data packets stored in MySQL database, consequently the loss packets can be calculated according to the sampling frequency, and the results shows in table 2 . Table 2 Data packets loss at different mobility status

\begin{tabular}{ccc|cc|cc|cc}
\hline \multirow{2}{*}{$\begin{array}{c}\text { Parameter } \\
\text { type }\end{array}$} & \multirow{2}{*}{$\begin{array}{c}\text { Mobility } \\
\text { status[m/s] }\end{array}$} & $\begin{array}{c}\text { Sampling } \\
\text { frequency[Hz] }\end{array}$ & \multicolumn{2}{|c}{ 1min } & \multicolumn{2}{c}{ 2min } & \multicolumn{2}{c}{ 5min } \\
\cline { 4 - 8 } & & & received & $\begin{array}{c}\text { Packets } \\
\text { loss }\end{array}$ & $\begin{array}{c}\text { Packets } \\
\text { received }\end{array}$ & $\begin{array}{c}\text { Packets } \\
\text { loss }\end{array}$ & $\begin{array}{c}\text { Packets } \\
\text { received }\end{array}$ & $\begin{array}{c}\text { Packets } \\
\text { loss }\end{array}$ \\
\hline \multirow{2}{*}{ ECG } & 0 & 200 & 11878 & 122 & 23663 & 237 & 59402 & 598 \\
& 1.5 & 200 & 11593 & 407 & 23245 & 825 & 57942 & 2058 \\
& 3 & 200 & 11328 & 672 & 22605 & 1395 & 56619 & 8831 \\
\hline \multirow{2}{*}{ Body } & 0 & 1 & 60 & 0 & 119 & 1 & 299 & 1 \\
temperature & 1.5 & 1 & 59 & 1 & 118 & 2 & 296 & 4 \\
& 3 & 1 & 58 & 2 & 116 & 4 & 293 & 7 \\
\hline
\end{tabular}

Fig.5 exhibits the average data packets loss rate at different mobility status. In each scenario we can get three data packets loss rates according to the three kinds of keeping time respectively. And the average data packets loss rate is the average value of these three loss rates. It is obviously that the average loss rate increases with the increase of the moving speed, while the overall packets loss rate is less than $6 \%$ which shows a superior stable performance under the different mobile status. This mainly thanks to the selection of ZigBee wireless communication mode of the WBANs nodes. In extreme cases, even though the ZigBee node disassociates with the ZigBee coordinator temporarily, it will try to connect to the coordinator automatically. Therefore, the stability can be guaranteed under the mobile status.

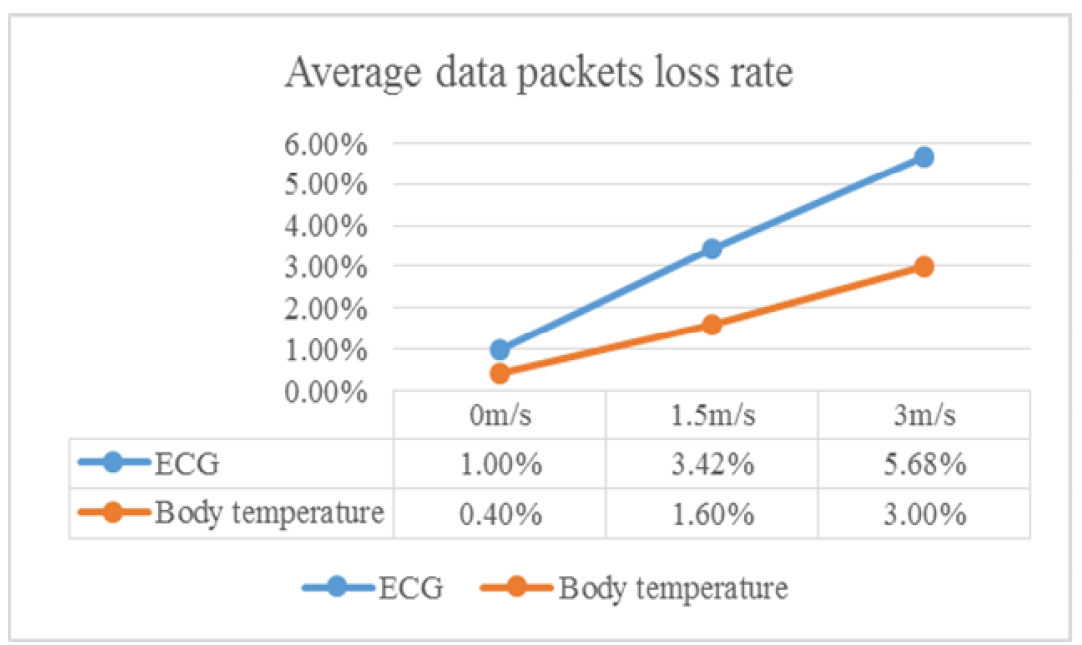

Fig.5 Average data packets loss rate at different mobility status

In app test, there are two prerequisites to note, one is the user has used our system for more than 1 minute, another is the user registered previously, and his/her basic information have stored in the database of the remote cloud server.

When meet the above requirements, we can test the android app. Fig.6 gives the test results. Fig.6(a) shows the main user interface (UI), here user can choose service according to personal conditions. Fig.6(b) and Fig.6(c) give the ECG result and the body temperature result, in this part, all 
the data is the latest and comes from the remote cloud server. Fig.6(d) gives the health report which is obtained according to the diagnosis of the current data by the diagnostic rules. The validation results show that the app works well, and with this app, the users or their families can conveniently to view the users' health status which greatly improve the practicability of the system.

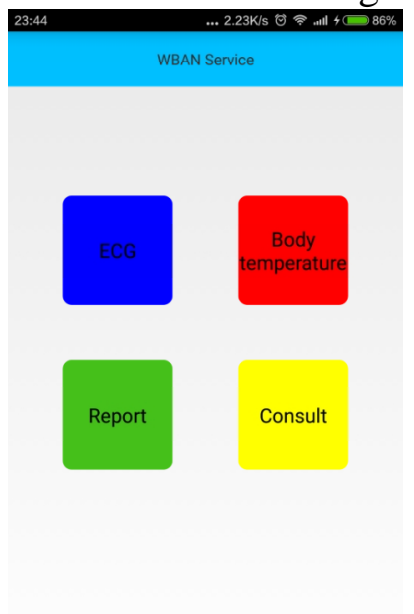

(a)

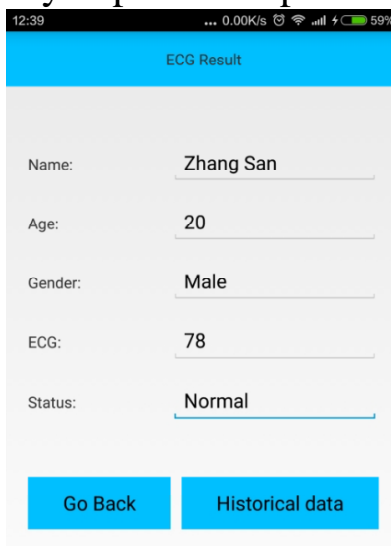

(b)

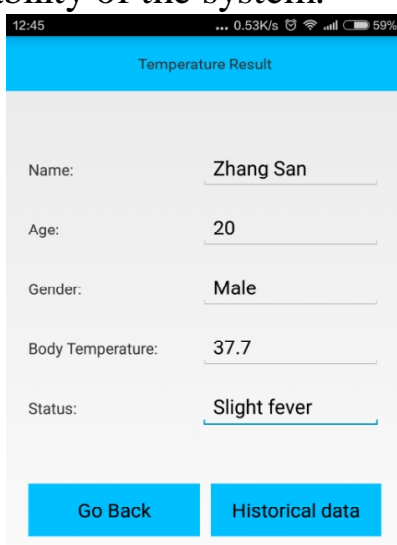

(c)

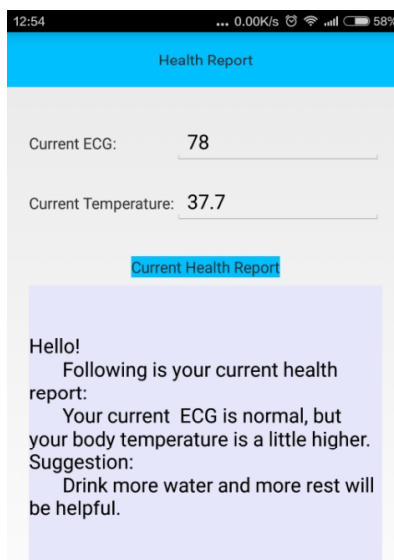

Go Back

(d)

Fig.6 Android app: (a) Main UI, (b) ECG UI, (c) Body temperature UI, (d) Health report UI

\section{Conclusions}

In this paper, we propose a cloud-service home health monitoring system based on WBANs for tracking the health status of the older people in real time while supporting their mobility. The system consists of a WBANs physiological parameters acquisition system and a remote cloud server. The specific implementation of WBANs physiological parameters acquisition system and the remote cloud server are described in detail. In addition, to facilitate the older people or their families to learn their health status, a simple android app is developed. Finally, mobility test and app test are carried out and the results show a superior performance of the proposed system.

\section{Acknowledgements}

This paper was supported by the Research Foundation of Science and Technology Commission of Shanghai under Grant No. 10DZ1500200, the Natural Science Fund of China (NSFC) under Grant Nos. 50975088, 51275173, and 51575186, the Fundamental Research Funds for the Central Universities under Grant No. WH0913009, Shanghai Pujiang Program under Grant No. PJ201000353, and Shanghai Software and IC industry Development Special Fund under Grant No. 120493.

\section{References}

[1] Latr'e, B., Braem, B., Moerman, I., Blondia, C., Demeester, P., A survey on wireless body area networks. Wireless. Netw 17(1):1-18, (2011)

[2] Ullah, S., Higgins, H., Braem, B., Latre, B., Blondia, C., Moerman, I., Saleem, S., Rahman, Z., Kwak, K.S., A comprehensive survey of wireless body area networks. J. Med. Syst. 36(3):1065-1094, 2012.

[3] Ragesh.G.K, Dr.K.Bashkaran. A survey on futuristic health care system: WBANs. Peocedia Engineering 30(2012) 889-896.

[4] Hande A, Cem E. Wireless sensor networks for healthcare: A survey. Computer Networks 54 (2010) 2688-2710

[5] Ahnn, J.H., and Potkonjak, M., mhealthmon: Toward energy efficient and distributed mobile health monitoring using parallel offloading. J. Med. Syst. 37(5):1-11, (2013)

[6] Diallo, O., Rodrigues, J.J., Sene, M., Niu, J.: Real-time query processing optimization for cloud-based wireless body area networks. Information Sciences, (2014) 